\title{
Correcting the Record - Cofactor binding of Human Pyrroline-5-Carboxylate Reductase
}

Kurt L Krause $^{1}$, Emily M Christensen ${ }^{2}$, Sagar M Patel ${ }^{3}$, David A Korasick ${ }^{2}$, Ashley C Campbell ${ }^{2}$, Donald F Becker ${ }^{3}$, John J Tanner ${ }^{2}$ ${ }^{1}$ Biochemistry, Otago University, Dunedin, New Zealand, ${ }^{2}$ Department of Chemistry, University of Missouri-Columbia, Columbia, United States, ${ }^{3}$ Department of Biochemistry and Redox Biology Center, University of Nebraska-Lincoln, Lincoln, United States E-mail: kurt.krause@otago.ac.nz

Pyrroline-5-carboxylate reductase (P5CR) is a key enzyme in proline metabolism that is found in both microbes and people. The amino acid proline has had a resurgence of interest because of its role in several medically related conditions. For example, it has been implicated in rare genetic conditions like cutis laxa, but it also has a role in in several human cancers.

P5CR reduces pyrroline-5-carboxylate utilizing either NADH or NADPH as a co-factor. The crystal structure, which has been solved from both bacteria and humans, is decamer composed of a dimer of pentameters. Each monomer from this symmetric complex has two domains, one of which is a Rossmann fold.

In 2006 the structure of the human enzyme was first reported (Meng et al., 2006) and described in detail. In this report the dinucleotide substrates, NADH and NADPH, were described as binding at a site more than $25 \AA$ away from the canonical Rossman fold ligand binding site. Further, this new binding site location did not match that of two bacterial P5CR structures reported in 2005 (Nocek et al., 2005)

As a result, we reinvestigated the heterodox dinucleotide binding site for human P5CR enzyme in two ways. First, we studied the co-factor binding sites in the original crystal structure using density from the EDS server. Next, we used PDBREDO to re-refine the complex. Finally, we calculated simulated annealing omit maps using PHENIX. All these results were compared to the previously published work, and our analysis suggested that the original location of the co-factor as reported in 2006 was not well supported.

We next purified, crystallized and re-solved the structure of the human enzyme and its complex with NADPH. In these new structures, the dinucleotide substrate NADPH binds exactly as expected, based on the historical binding locations found in other NADPH binding enzymes, as well as in the 2005 bacterial P5CR crystal structures.

Misplaced ligand binding and misrefined crystal structures have recently been reported by multiple authors. Good suggestions have been made on how to avoid the most common errors. We recommend adding an assessment of the canonical ligand location (CLL), including a superposition and RMS deviation compared to similar enzymes, to the set of ligand vetting tools.

Christensen, et al., (2017) J. Biol. Chem., in press.

Meng, et al., (2006) J. Mol. Biol. 359, 1364-1377

Nocek, et al., (2005) J. Mol. Biol. 354, 91-106

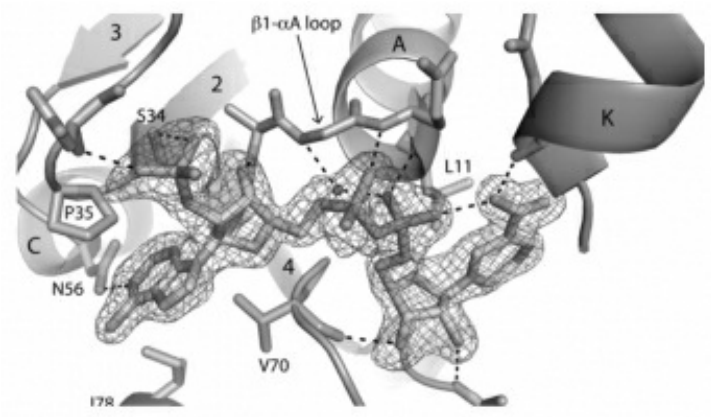

Keywords: Protein-Ligand binding, NADPH binding, Ligand validation 